\title{
La precaución como principio de acción sostenible
}

\author{
Precaution as a principle of sustainable action
}

\author{
ANDONI EIZAGIRRE
}

Universidad de Mondragón

RESUMEN. Este trabajo comprende el principio de precaución como indicador de las tensiones en los estándares de gobierno. El principio de precaución no es una medida provisional y preventiva tomada por la falta de conocimientos y causado por la magnitud de los riesgos; no es una variante del análisis del riesgo. Antes bien, es un principio que genera reglas y normas orientadas a identificar espacios de incertidumbre y debatir acerca de lo que denominamos conocimiento relevante.

Palabras clave: Incertidumbre, regulación, análisis del riesgo, principio de precaución.

\section{Introducción}

Las implicaciones operativas del principio de precaución han marcado parte de las recientes controversias en torno a las políticas de regulación de la ciencia y la tecnología. Mi hipótesis de trabajo es que las distintas interpretaciones relativas al principio de precaución son motivadas por las diferentes maneras de entender los estándares de regulación y en general la ciencia reguladora.

Cabe precisar que la Unión Europea también ha reconocido la necesidad de adaptar el análisis tradicional del riesgo e incorporar al proceso de evaluación normas de acción dirigidas a integrar las incertidumbres motivadas por el avance del
ABSTRACT. In this paper I propose that the precautionary principle is an indicator of the tensions in standards of regulation. The precautionary principle is not a provisional and preventive measure that is decided because we do not have a knowledge adapted to determine the risks; precaution is not a variant of risk analysis. The precautionary principle promotes standards and norms to identify areas of uncertainty and debate on relevant knowledge.

Key words: Uncertainty, regulation, risk analysis, precautionary principle.

conocimiento científico. De alguna manera se contempla la idoneidad de situar el problema en el marco de los estándares de regulación, lo que da a entender que los análisis del riesgo han de ser completados por nuevas formas de gobernar los impactos del avance científico-técnico.

En lo que a la estructura de mi trabajo se refiere, en el segundo apartado se identifican sucintamente las diferentes posturas sobre el principio de precaución. Esto me servirá para avanzar mi diagnóstico y el marco analítico sobre la problemática, a saber: el principio de precaución indica un modo alternativo de entender la actividad reguladora. Los conflictos surgidos en torno al principio de precaución — como se aborda en el 
tercer apartado - pueden ser un indicador de la tensión entre las diferentes maneras de entender el problema del riesgo y el criterio de evidencia, y no tanto una decisión preventiva y políticamente motivada por los límites del conocimiento disponible. En ese caso, el debate sobre el principio de precaución es reflejo de un debate que concierne más bien a los aspectos que los elementos y mecanismos de regulación deben incorporar en sus políticas de gobernar la ciencia y la tecnología.

\section{La emergencia de la precaución y sus interpretaciones}

Los informes institucionales referentes al principio de precaución se han extendido en un tiempo relativamente breve a diferentes ámbitos de la ciencia y la sociedad; cierto es que ya en la década de los setenta se mencionaba la precaución en las políticas europeas ambientales, asumiendo que los gobiernos pueden mitigar y evitar daños en el ambiente e integrando a su vez no sólo indicadores económicos en los procesos de innovación, pero en cualquier caso ha adquirido notoriedad recientemente a través de los tratados y declaraciones internacionales.

En mi caso, sin ánimo de exhaustividad, voy a presentar brevemente tres posturas principales, que a su vez vienen a ser tres maneras diferentes de concebir la precaución. En la parte final, indagaré una crítica de los supuestos de la cultura política de la ciencia dominante que será desarrollada y ampliada en el tercer apartado.

\section{a. La interpretación crítica: evidencia cientifica e individualidad}

Hay una interpretación según la cual la precaución es una estrategia que traba y pretende imposibilitar el proceso de innovación, declara inaceptable proclamar la «seguridad» y «falta de riesgo» como condición de posibilidad del proceso de innovación, en tanto que los riesgos son constitutivos a las tecnologías y la innovación debe guiarse por los criterios de utilidad y perjuicio grave, y en todo caso opina que debería corresponder a la parte opositora probar los riesgos que afirma tiene la tecnología en cuestión. Con las nuevas tecnologías no hay motivo alguno para revolucionar la lógica y los estándares de prueba; la condición de seguridad absoluta es reflejo de la aversión al riesgo («risk aversion») y el desprecio a las capacidades de la agencia humana. La defensa del ambiente natural - que dicen hacer los movimientos ecologistas - es el sustituto funcional de la pérdida de confianza en las instituciones sociales y políticas, proporcional al desprecio de la condición humana y alimentada por los medios de comunicación de una manera sensacionalista, que ha propiciado en algunos países su justificación para encubrir medidas proteccionistas vedadas por los acuerdos internacionales sobre el comercio.

Esta posición crítica, principalmente erradicada en los Estados Unidos, se consolida como respuesta a la moratoria europea a los organismos genéticamente modificados aprobada en base a la incertidumbre que implica su aplicación y comercialización; considerándola ilegal, se presenta un recurso contra la Unión Europea ante la Organización Mundial del Comercio en el año 2003. ${ }^{1}$ Además, según esta postura, existen medidas reguladoras aptas e idóneas en el comercio internacional para la protección de la salud y el ambiente ("sanitary and phytosanitary measures»), establecidas en base a dos principios: la evidencia científica de los riesgos (" sufficient scientific evidence»), y el carácter temporal (de la prevención) que sin mayor dilación ha de ser vencida. ${ }^{2}$ 
Es así como la precaución descuida la lógica tradicional, en tanto que: (a) acuerda para todos los productos una medida reguladora uniforme (no según las características de cada producto, sino que basándose en el método del desarrollo del producto); (b) proclama la seguridad y además sin considerar la evidencia científica del riesgo; (c) acota políticamente el nivel de aceptación del riesgo; (d) se impone la máxima "guilty until proven innocent» frente a la tradicional «innocent until proven guilty» (Hathcock, 2000). Las bases filosóficas de la precaución también resultarían ser contradictorias: en tanto no podemos prever los impactos potenciales, se consideran catastróficas e irreversibles, y entre tanto la viabilidad debe garantizar la certidumbre absoluta de su seguridad.

Esta paradoja más bien prueba — según los críticos del principio- que cuando menos la negociación con las incertidumbres científicas es problemática y está comprendida en otras contradicciones: ¿existe una seguridad científica total?; una vez decidida la prevención a tomar, ¿necesitaremos un nivel mínimo de conocimiento para demostrar esa legitimidad?; cuando manifestamos la determinación de prevenir el impacto, ¿seremos capaces de prevenirlo en la medida en que no conocemos la relación causa-efecto? En definitiva, para los críticos esas lagunas y carencias resultan suficientes para esquivar la peculiaridad de la precaución. Según esta perspectiva, los defensores del principio se contradicen por sí mismos: al tiempo que toman como pretexto la incertidumbre y la complejidad de los efectos, se presentan posiciones unívocas, es decir, se anticipa y escenifica la catástrofe, pero sin un conocimiento seguro de la misma. Con todo, además de la aplicación del principio de precaución y sus consecuencias, las condiciones de demostración de su legitimidad permanecen muy difuminadas. ${ }^{3}$

\section{b. La interpretación flexible: incertidumbre y moratoria}

Acabamos de ver que, según la interpretación crítica del principio de precaución, emerge un cambio en la lógica de la regulación, motivado por tres razones: el principio de precaución subestima la evaluación de la evidencia científica, es una herramienta que corresponde al ámbito político, y más que en los cálculos determinista y probabilista, se basa en las expectativas («expectations») sobre los impactos (Holm y Harris, 1999, 398). Una segunda postura alternativa asume que a menudo existen dificultades para percibir las incertidumbres y los impactos a largo plazo de una tecnología, agregando que no por ello el proceso de regulación debe estar condicionado por las preocupaciones psicológicas, el valor mediático concedido y las tendencias socioculturales dominantes. Por este motivo, proponen una moratoria para obtener nuevo conocimiento y acordar la viabilidad del proyecto; los límites del conocimiento disponible dificulta la medición de las probabilidades y magnitudes del impacto, lo que compromete a adquirir las pruebas, evidencias e información solicitadas en un plazo acordado, para a continuación tomar una decisión (política) a partir de las evaluaciones (científicas) favorables y/o contrarias.

Es una postura que claramente representa la Unión Europea, motivada por una serie de cuestiones en parte antagónicas como son la pérdida de confianza social hacia las instituciones, la emergencia de las incertidumbres científicas y el rol de la innovación en la productividad (CEC, 2000, 8). Debemos recordar que haciendo caso omiso a las palabras de la comisaria de Medio Ambiente de la Unión Europea, Margot Wallstrom ( $« \mathrm{He}$ mos esperado demasiado tiempo antes de actuar. La moratoria es ilegal y no se 
puede justificar... el alcance de la biotecnología ha sido pobremente apreciado en Europa», 13 de julio de 2000), los gobiernos de Austria, Francia, Alemania, Italia, Grecia y Luxemburgo prohibieron los productos modificados genéticamente que la Comisión había aprobado. ${ }^{4}$

Para una comprensión cabal de esta postura (CEC, 2000), corresponde reflexionar con atención el significado que tiene la integración de la incertidumbre al análisis de los impactos negativos. Es cierto que la gestión se centra entre la prohibición y la moratoria, pero esto se debe a que integra en el proceso de evaluación el ambiente natural y la salud humana, los efectos negativos y las incertidumbres que presuponen el desarrollo y la aplicación del producto novedoso. La precaución en este sentido responde a tres razones (véanse: Jordan, 1999, 2005): se reconoce la incertidumbre en la gestión de los recursos naturales; se considera que la prevención de amenazas es más propicia que la reparación de los daños; y se le da tanta importancia al tratamiento del riesgo como a la seguridad.

Éste es el dilema al que se enfrentan los políticos europeos: contraponer y convenir un equilibrio entre los riesgos que pueda suponer el impacto tecnológico en el ambiente y en la salud y la libertad económica de la industria y la empresa. Ciertamente, la información científica y estadística se valen del análisis del riesgo de tales situaciones, a través de la integración en un proceso programado de evaluación, gestión y comunicación del riesgo. El contexto de decisión y las medidas de prevención, sin embargo, no siempre se llevan a cabo con la información científica suficiente. Por este motivo, la justificación del principio de precaución puede estar motivada por dos razones: por un lado, porque la información científica es insuficiente, o no resulta completa para la toma de una decisión; $y$, por otro lado, porque existen indicios de que pueden suceder efectos adversos en el ambiente y la salud humana, o que las medidas de protección existentes no son las adecuadas.

El principio de precaución, en la Unión Europea, debe considerarse dentro de un marco estructurado del análisis del riesgo. Son tres los elementos principales: la evaluación del riesgo (fase científica), la gestión del riesgo (fase política) y la comunicación del riesgo (fase social), sucesivamente. El principio de precaución se sitúa en la fase política de la gestión del riesgo. A efectos de justificar esta posición, se realizan las siguientes precisiones (CEC, 2000, 3-4): por un lado, hay que distinguir el principio de precaución que los políticos utilizan para la gestión del riesgo y la precaución que los científicos han venido aplicando y aplican en los datos científicos utilizados para la evaluación; por otro lado, para utilizar el principio de precaución en la gestión del riesgo, tiene que cumplirse previamente la siguiente condición: que los científicos hayan identificado los efectos peligrosos de un fenómeno, producto o proceso, y que la evaluación científica no pueda determinar el riesgo con seguridad suficiente; en tercer lugar, las aplicaciones de un planteamiento basadas en el principio de precaución han de partir de una evaluación científica y, si fuera posible, de identificar en cada fase de identificación, estimación y valoración el nivel de incertidumbre científica correspondiente.

La Unión Europea enfatiza la importancia de realizar correctamente esa separación analítica, normativa y política en el análisis del riesgo (CEC, 2000, 13). En realidad, el principio de precaución surge motivado por dos razones principales (véase Godard, 1997). En primer lugar, hay un cambio en el modelo de evaluación. Se replantea la calidad de los productos, debido 
a los riesgos que puedan suponer en el ambiente y la salud las consecuencias de la producción de alimentos y mercancías; por de pronto no se considera adecuado diferenciar los productos novedosos y los procedimientos seguidos; no solamente se realiza el análisis de un producto para detectar los riesgos y estimar su calidad, sino que se integran también los datos relativos a la calidad del proceso productivo: así, se recomienda evaluar también los ciclos de vida del producto, los certificados de la gestión ambiental y los procedimientos de seguimiento. En cuanto al segundo motivo, se revisan las teorías clásicas sobre el libre mercado, en la medida en que aquellas infravaloran la problemática del ambiente y la gestión de los riesgos («externalities»), y ahora no se da por hecho que el productor integre el coste social de un producto, por el contrario, también se integran en el análisis de viabilidad las consideraciones relativas al ambiente y bienestar social. Es en este contexto en el que habría que situar la postura europea sobre el principio de precaución (véase Sanderson y Petersen, 2002).

c. La interpretación fuerte: irreversibilidad y alternativas

En torno a las interpretaciones sobre el principio de precaución, cabe poner de relieve en tercer lugar la posición fuerte («strong version»), que tiene dos peculiaridades a destacar: (a) la certidumbre absoluta sobre ausencia de impactos negativos como condición de posibilidad de la aplicación tecnológica y (b) la carga de la prueba interpuesta a los promotores de la investigación y aplicación del producto novedoso; entre tanto, incluso a falta de evidencias que determinen la relación entre causa y efecto, esta posición reclama la prohibición y la investigación de nuevas alternativas tecnológicas. Esta manera de obrar tiene como causa que los ámbitos de incertidumbre provocados por los nuevos conocimientos y los riesgos de las nuevas tecnologías son cada vez mayores, de tal manera que las anticipaciones son más escasas a la vez que las consecuencias irreversibles. ${ }^{5}$

La declaración «Wingspread Statement on the Precautionary Principle», firmada entre los días 23 y 25 de enero del año 1998 con motivo de las conferencias organizadas sobre la materia, es la referencia de partida de los defensores de la interpretación fuerte del principio de precaución. Allí se deduce que la difusión y utilización de productos tóxicos, la explotación de los recursos y las alteraciones físicas del ambiente han tenido consecuencias imprevistas e indeseadas en la salud humana y en el ambiente; " algunas de estas preocupaciones son las altas tasas de dificultades de aprendizaje, el asma, el cáncer, las malformaciones letales y las especies en extinción; junto con el cambio climático global, la disminución del ozono estratosférico y la contaminación mundial con substancias tóxicas materiales nucleares». Además, se da constancia de un hecho adicional, a saber: las regulaciones ambientales $y$ otras decisiones similares, como el análisis técnico del riesgo («risk analysis»), no han ratificado ninguna aptitud para la protección de la salud y ambiente. En la misma medida, se insiste en que es incalculable el riesgo tecnológico que amenaza a los seres humanos y el ambiente, y que en consecuencia resulta fundamental la promoción de nuevas medidas para regular las acciones humanas. La declaración articula su propuesta a partir de tres motivos esenciales: porque las alteraciones naturales derivadas de las acciones humanas tienen consecuencias negativas en la salud y en el ambiente; por la falta de efectividad de los mecanismos de regulación existentes; y, por las dimensiones incalculables, catastróficas e irreversibles del riesgo tecnológico. ${ }^{6}$ 
Así que el punto de vista desde el cual se considera y se analiza esta perspectiva incorpora las reflexiones asociadas a los riesgos ambientales y sociales que abarca y complica la acción humana. Esta perspectiva articula y justifica la implementación del principio de precaución conforme a cuatro premisas. En primer lugar, las medidas precautorias han de tomarse sin dilación cuando una actividad puede amenazar la salud humana o el ambiente natural, incluso cuando no haya manera de establecer las relaciones causa-efecto de un modo científico. En segundo lugar, la carga de la prueba («the burden of proof») recae sobre quien propone la actividad y debe probar la evidencia de ausencia del riesgo; la ausencia de evidencia de riesgo ratificaría la anticipación de catástrofes y la asimetría entre intensidad innovadora y su regulación. Como tercera peculiaridad, el proceso de aplicación del principio de precaución debe ser transparente y democrático, y debe incorporar a las partes potencialmente afectadas. Por último, se deben identificar y discutir las distintas alternativas de ese proceso, incluida la noacción.

La interpretación fuerte del principio de precaución presupone y enfatiza que nuestras aptitudes tecnológicas y las consecuencias potenciales de la actividad humana superan nuestro nivel de previsión y conocimiento. Los impactos además resultan irreversibles y así es como toma sentido el imperativo moral adjunto a esta tercera interpretación del principio de precaución, es decir, que la humanidad no ha de poner en riesgo su existencia y supervivencia a cambio de apostar por los avances tecnológicos. 7

\section{d. Precaución y prevención}

Las normas basadas en la ciencia son objeto de competencia política en su propósito de concretar los estándares de regulación y aprobar la comercialización de nuevos productos. En lo que sigue propongo incorporar aquella constatación y ampliar su reflexión a los principios de acción en ciencia y tecnología, y no acotar la discusión sobre la precaución al uso político del análisis del riesgo, como parece comprenderse en las críticas mutuas entre las diferentes interpretaciones sobre el principio de precaución.

Como acabamos de ver, los partidarios de la producción y comercialización de todo avance tecnológico enfatizan que las normas decisorias han de estar basadas en la ciencia. Esta postura presupone cuatro supuestos (Kerr, 2003, 89): el consenso cognitivo y metodológico; la ciencia libre de valores; el conocimiento disponible como garantía para formular una hipótesis científica y evaluar el riesgo; y el apoyo popular de las desiciones sobre ciencia y tecnología. Si nos referimos a la Unión Europea, en este caso también se admiten las normas y regulaciones de base científica, pero se constata que la última decisión ha de ser política. Así, en aquellos casos en que existe incertidumbre científica, la decisión ha de ser política y se deben incorporar factores sociales y económicos más amplios. Por su parte, la posición fuerte no rechaza la ciencia fundamentada, pero cuestiona por el contrario la manera dominante en que se entiende. Al igual que la Unión Europea, enfatiza las experiencias históricas y los distintos problemas hallados en el análisis del riesgo relativos a las incertidumbres técnicas, epistemológicas y metodológicas; así por ejemplo, emergen problemas de comprensión científica sobre las propiedades de un alimento genéticamente modificado, e incluso dificultades para detectar e identificar los efectos, y siempre cabe cuestionar los valores que incorporan los científicos en la evaluación de los riesgos, quedando al descubierto los problemas del sistema estándar de regulación. 
Sin embargo, las discusiones no han abordado una serie de cuestiones de relevancia. Entre esas cuestiones caben destacar: los supuestos y valores metodológicos destinados a clausurar los riesgos e incertidumbres; la función que cumplen los sistemas epistémicos tanto en el desarrollo científico-técnico y social como en la constitución del orden natural y social; los contextos de incertidumbre que agravan los nuevos conocimientos; la función de lo teórico en la investigación científica y el modo como se acepta el conocimiento relevante en las prácticas científicas.

Antes bien, las controversias han reproducido la demarcación clásica entre ciencia y política (ciencia fundamentada $v s$. valores morales). Y su transposición normativa al ámbito político, acarrea la confusión generalizada en torno al alcance del principio, así por ejemplo el repertorio europeo asociado a la determinación concibe la precaución como una variante excepcional del análisis del riesgo y por tanto legitima la prevención en contextos de incertidumbre e ignorancia para prevenir daños no deseados. Es así como se confunden la precaución y la prevención, limitando la deliberación política a la conformidad de gestionar la innovación de manera preventiva.

Por el contrario, en el tercer apartado defiendo que el principio de precaución no es una medida provisional y preventiva tomada por la falta de conocimientos y causado por la magnitud de los riesgos; no es una variante del análisis del riesgo. Antes bien, es un principio que genera reglas y normas orientadas a identificar espacios de incertidumbre y debatir acerca de lo que denominamos conocimiento relevante; aquello que el análisis del riesgo da por sentado, el principio de precaución lo propone como objeto de competencia política y social. Es así como opera simultáneamente con el polo objetivosocial, se constituye a través de una compleja imbricación entre elementos heterogéneos y evidencia que los conflictos sobre los estándares de regulación pueden ser también conflictos sobre estilos de hacer ciencia y sobre modos de entender la ciencia como algo socialmente relevante. Por ello, se plantea que es el modo de comprender la cultura reguladora la que condiciona el debate sobre el principio de precaución; es posible que un análisis sofisticado de la ciencia reguladora borre las fronteras tradicionales entre evaluación-gestión, ciencia-política $\mathrm{y}$, debate cognitivo-debate resolutivo. La precaución no puede utilizarse como mero mecanismo político, pero la evaluación del riesgo tampoco es una mera práctica posibilitada por criterios y evidencias científicas. El tercer apartado presenta y defiende (la hipótesis «precautionary uncertainty») que no es una situación de incertidumbre la que justifica la precaución, al contrario, es una regulación prudente la que nos motiva a evaluar de una manera integral las variables que la aproximación tradicional del riesgo infravalora. La precaución no determina lo que debemos hacer, pero nos puede ofrecer la oportunidad de replantearnos a qué debemos poner atención, de tal manera que puede generar principios de acción diferentes, en base a las reglas, procedimientos, criterios y preocupaciones que se incorporan en los mecanismos de producción, uso y validación del conocimiento. ${ }^{8}$

\section{Tensiones en los estándares de regulación}

Las controversias en torno a las nuevas tecnologías abren un amplio abanico de cuestiones, tienen un carácter horizontal y están sujetas a implicaciones diversas. En este sentido, quiero plantear la precaución como principio pragmático en la acción científica y tecnológica que amplía el proceso de regulación, a la vez que 
se hace cargo de la revisión del consenso cognitivo y metodológico que presupone la ciencia académica. Esta crítica puede servir para revisar lo que entendemos por ciencia reguladora y por consiguiente el valor de la precaución como principio-guía de las políticas científicas y tecnológicas. Ciertamente, desde el preciso momento en que se debaten las cuestiones a considerar en el proceso de evaluación, esto permite ampliar el debate también a las alternativas tecnológicas, líneas de innovación, modelos de vida y ámbitos de participación.

Para su comprensión, debemos recordar que en las premisas epistemológicas dominantes los sistemas ambientales resultan relativamente estables y conocibles; las disciplinas, conceptos y análisis, y las consiguientes definiciones, los métodos y los indicadores pueden variar, no obstante, los problemas ambientales son definidos en términos técnicos y las soluciones tienen un carácter resoluble por científicos, ingenieros y/o economistas; el modo de gestionar el problema resulta propicio a la política nacional, el negocio empresarial y la coordinación entre ambas a nivel local e internacional. En definitiva, el juego de suma cero puede ser reemplazado por un lenguaje positivo a través de un cambio ambientalmente orientado y compatible con el crecimiento económico. De esta manera, las instituciones sostienen el crecimiento económico y la protección ambiental no sólo como compatibles sino mutuamente dependientes.

En lo que sigue, se abren tres líneas de reflexión: (a) se replantea el concepto ciencia reguladora, (b) se propone una hipótesis alternativa del principio de precaución y, por último, (c) se revisan las premisas epistemológicas y el alcance limitado de los reproches que realizan las diferentes instituciones en torno a los estándares de regulación.

\section{a. Ciencia reguladora y principio de precaución}

En el ámbito de la Unión Europea, la legislación y actividad reguladora han sido modificadas con la emergencia y consolidación del principio de precaución, lo que ha reavivado un conflicto de carácter político entre los EEUU y la UE. A mi parecer, sin embargo, es demasiado audaz concluir que el conflicto responde a dos modos divergentes de concebir la actividad reguladora. Por el contrario, un análisis más detallado de las diferencias y semejanzas es probable que nos avance pistas para una interpretación alternativa del conflicto existente.

En la Unión Europea, las reformas institucionales y los procesos de convergencia han supuesto el conflicto entre modelos de entender la ciencia, los expertos y los estándares de regulación. Al negociar con estos conflictos, la precaución y la incertidumbre científica han resultado ser los elementos más controvertidos, en el que sobresalen los problemas de la ciencia fundamentada como garantía de la regulación, se cuestiona la imagen corriente de la ciencia, al reconocer que los estilos de razonamiento y los criterios de satisfacción son un producto histórico y colectivo, y se constatan las dificultades de establecer una diferencia analítica y normativa entre ciencia del riesgo y su percepción social, así que recobra relevancia la disputa sobre las bases legales e instrumentos analíticos relativos al análisis del riesgo. Pero todavía más, debido a los problemas de legitimación del sistema de ciencia a nivel europeo («déficit de confianza») y el nuevo repertorio asociado a la transparencia, la participación y el diálogo con la sociedad civil, la incorporación del principio de precaución obliga y permite la armonización de la actividad reguladora de la Unión Europea, guiada por una política 
basada en la ciencia ( science-based policy») que a la vez garantiza y promueve la aceptabilidad social.

A partir de estas consideraciones, bien podríamos comprender las discrepancias creadas a ambos lados del océano, pero un análisis más detallado también nos avanza algunas ideas más proclives a destacar los puntos de unión. El marco institucional europeo tiene dos características importantes: da prioridad a la evidencia científica para tomar decisiones políticas y enfatiza la importancia de las consideraciones sociales. La evolución tecnológica para su validación contiene aspectos socio-técnicos que la regulación tradicional no estima, ahora se juzga que la regulación del riesgo debe ser una expresión de los deseos sociales, garantizando que los desarrollos tecnológicos y las decisiones de mercado cumplan con las prioridades de los consumidores de un modo seguro. La ciencia reguladora ha de promocionar las alternativas comerciales de las nuevas tecnologías, pero reconociendo también el principio de precaución y las consideraciones éticas para así posibilitar la confianza de los consumidores. De esta forma, por lo tanto, el marco institucional se organiza de la siguiente manera: las tecnologías se evalúan en el interior de la comunidad de expertos, pero en el momento de la gestión se debe considerar el principio de precaución, lo que permite que tras el proceso de evaluación se integren en el proceso de gestión las diferentes preocupaciones éticas y sociales existentes.

Esto da a entender que - también a nivel europeo - los estándares de regulación se organizan como respuesta a los riesgos técnicos, pero ignoran nuevamente los resultados que las percepciones sociales sobre el riesgo (límites del conocimiento científico y su omisión en la comunicación pública; convergencia de intereses; efectos de segundo orden en la naturaleza y la sociedad) y los problemas epistemológicos que se han venido constatando en las últimas décadas. La perspectiva dominante indica la hegemonía de una cultura política de la ciencia, considerando exclusivamente el riesgo (biofísico) e infravalorando las percepciones ciudadanas y sus variables explicativas.

Si centramos nuestro análisis -en este trabajo - en los problemas que contiene la evaluación de las probabilidades y magnitudes del riesgo (Renn y Klinke, 2001), éstas se realizan con variables conocidas y el conocimiento disponible; su objetivo es determinar las consecuencias de una práctica tecnológica, aunque a menudo presente las consecuencias como probabilidades. En consecuencia, en la evaluación del riesgo la ciencia nos ofrece aquel conocimiento útil para la identificación y valoración de los problemas. Pero esto deberíamos entenderlo con cierta prudencia. De hecho, el conocimiento científico puede limitarse a la identificación de unos determinados problemas, es decir, la evaluación podría limitarse a la consideración de los elementos conocidos. Por este motivo, el procedimiento científico corre el riesgo de infravalorar otro tipo de elementos e incertidumbres. En la evaluación tradicional del riesgo se establece previamente la legitimación de las diferentes preguntas y preocupaciones. Y esto, además, determina las alternativas institucionales, políticas y culturales de una innovación tecnológica (ESRC, 2000a).

La ampliación de las funciones y responsabilidades de las políticas científicas y ambientales al ámbito de la regulación ha presupuesto el análisis del riesgo («risk analysis») como base científica y política para establecer la magnitud de los daños y las probabilidades del impacto. No obstante, la evaluación del riesgo ha tenido múltiples problemas (Jasanoff, 2003). Por un lado, la seguridad sólo pue- 
de obtenerse dentro de unos contextos determinados y limitados, en concreto, cuando las amenazas son evidentes y a corto plazo. Esto contiene graves implicaciones, es decir, los problemas para integrar las incertidumbres y complejidades con herramientas cuantificables y objetivas para la toma de decisiones «... puede llevar a catástrofes ambientales y crisis políticas que se manifiestan pausadamente pero con altos costos para la sociedad (...) Las zonas de alta incertidumbre deben ser reconocidas por los investigadores y los responsables políticos: la ausencia de evidencia de riesgos no es lo mismo que evidencia de ausencia» (ESRC, 2000b, 3). A pesar de que el análisis del riesgo prevé el conocimiento integral, existen dificultades para obtener un conocimiento fiable, las tecnologías son complejas, influyen infinidad de factores y las interacciones de éstos abre los campos de incertidumbre, desconocimiento e indeterminación (Lemkow, 2002; Wynne y Meyer, 1993). Así comprendemos que «los tradicionales métodos cientificos tratan de reducir la incertidumbre en lugar de representarla. Factores sobre los que nada sabemos son a menudo transformados en incertidumbres controlables y examinados con métodos probabilísticos» (ESRC, 2000b, 12).

En este sentido, en segundo lugar, la idea de conocimiento científico que presupone la imagen corriente se retrotrae a lo dado y sirve como justificación epistémica del análisis del riesgo, pero también tiene estos otros problemas (ESRC, 2000a, 2000b): omisión de las condiciones de gran subdeterminación; utilización excesiva de analogías y generalización de una evidencia con investigaciones selectivas; falta de coordinación de las investigaciones y disciplinas; e incluso aspectos más contextuales como el choque entre el tiempo científico y el so- cial, así como la credibilidad excesiva a las publicaciones en revistas de prestigio e infravaloración de los descubrimientos negativos que no llegan a publicarse.

Todo esto deja al descubierto los problemas que la cultura de la evaluación del riesgo tiene para la ciencia reguladora. Es decir, el único problema que pueda tener la ciencia reguladora al evaluar el riesgo no radica en la incertidumbre de las probabilidades de que ocurran los daños conocidos: además, el conocimiento también puede estar condicionado por las incertidumbres y la consiguiente dificultad de predecir los impactos. ${ }^{9} \mathrm{El}$ único origen de los problemas por tanto no radica en la carencia de datos relevantes o en la falta de acuerdo para la interpretación de estos datos.

En tercer lugar, los valores no-científicos se integran en los procesos de evaluación del riesgo. Por ejemplo, la decisión de los científicos también desempeña una función social en la definición del criterio de evidencia, la identificación del tipo de amenaza a investigar, la negociación del grado de aceptabilidad de un riesgo, o en lo relativo a los instrumentos para la gestión de riesgos. ${ }^{10} \mathrm{~A}$ pesar de que los problemas mencionados pertenecen a niveles de crítica completamente diferentes, nos avanzan una manera más compleja de concebir la evidencia científica para la definición del riesgo, y urge asimismo reflexionar sobre las nociones de evidencia, control y certidumbre que presupone el análisis del riesgo.

Estas diferentes críticas resultan de momento suficientes para mi objetivo. Los científicos en sus prácticas siempre deben responder a cuestiones tales como: cuáles serán las cuestiones a analizar en el proceso de regulación; cuáles son los efectos causantes; qué se entiende por evidencia adecuada; o quién debe identificar, estimar y valorar las evidencias. Es importante considerar estas diferentes cuestio- 
nes que conciernen a la ciencia reguladora, no solamente para entender las discrepancias entre la UE y los EE.UU., sino también para comprender el valor del principio de precaución. Efectivamente, es una «evaluación política del riesgo» la que debe decidir sobre cuestiones como: la elección de los impactos que se analizan durante la evaluación; los tipos de evidencia que se integran al identificar, estimar y valorar los riesgos; la forma de interpretar la evidencia disponible; el modo de dar (una) respuesta a las incertidumbres; la definición de los diferentes tipos de evidencia necesarios o suficientes para sostener los distintos tipos de juicio; la elección del nivel de protección y tolerancia para establecer los grados de aceptabilidad social del riesgo y la incertidumbre. Son cuestiones que el análisis del riesgo da por hechas, y de esta manera los conflictos entre modelos institucionales (estadounidense $v s$. europeo) quedan condicionados al valor que conceden a consideraciones sociales y culturales en el proceso de gestión del riesgo que sigue al proceso de evaluación.

Los modos de entender la evaluación del riesgo y la actividad reguladora pueden condicionar la interpretación del principio de precaución; desde el momento que se acepta que en el proceso de regulación se integran valores epistemológicos, normativos y sociales, el principio de precaución no solamente avanza un modo de regular la ciencia sino que más bien replantea la relación que se establece entre las incertidumbres y su regulación. En otras palabras, según los marcos cognitivos, se da lugar a un conflicto de valores en torno a la incertidumbre científica; la incertidumbre no es necesariamente un accidente o excepción en la práctica científica que se supera con nuevos conocimientos.

En este sentido, el principio de precaución se puede valorar de manera dife- rente. Ciertamente, el principio de precaución se ha utilizado para justificar los problemas para calcular el riesgo y los límites del conocimiento disponible; al mismo tiempo, las diferentes interpretaciones adoptan una postura ante el principio y se sirven de ella para críticar los motivos políticos del oponente. De la misma manera, los debates sociales y morales deben tener lugar en el proceso posterior a la evaluación. No obstante, si reconocemos las críticas que ha merecido en los últimos años el análisis del riesgo y por extensión la ciencia reguladora, en ese caso podemos comprender de manera alternativa que la precaución «incorpora dos tipos de obligaciones normativas: una prudencial que trata de anticipar daños potenciales, y otra moral que trata de juzgar la adecuación del conocimiento disponible» (Levidow, Carr y Wield, 2005b, 263). Cierto es que el principio de precaución puede estar relacionada con la debilidad de la evaluación del riesgo tradicional, pero las críticas que hemos utilizado para identificar esas debilidades nos proporcionan también la oportunidad y ventaja de replantear el principio de precaución de manera diferente.

\section{b. Una relación alternativa entre precaución e incertidumbre}

Si el análisis del riesgo puede tener sentido en contextos de incertidumbre caracterizados como de «riesgo» e «incertidumbre», el principio de precaución puede resultar más adecuado en aquellos contextos en los que imperan la «ambigüedad»y la «ignorancia» (Mayer y Stirling, 2002). Aquí cabe la posibilidad de concebir el principio de precaución como una variante del análisis del riesgo; se propone avanzar en el desarrollo de nuevos conocimientos y mientras tanto reconocer la importancia de una gestión prudente (versión europea). Ahora bien, también se puede oponer la precaución 
como modelo alternativo a la evaluación tradicional del riesgo. De manera alternativa, se pueden proponer las siguientes estrategias: realizar una evaluación interdisciplinar; reconocer diferentes expertos y reclamaciones, para que tengan voz las diferentes preferencias, preocupaciones y conocimientos tácitos; garantizar la independencia de la evaluación e informar de los valores subjetivos cuando éstos intervienen; al realizar la evaluación de la viabilidad y utilidad, en vez de dar prioridad a los criterios de optimización económica, priorizar el criterio de resistencia. Sea como fuere, la evaluación probabilística del riesgo sólo puede utilizarse en unas determinadas situaciones.

Además, desde el momento que cuestionamos el valor de la evidencia científica en el proceso de evaluación del riesgo, no menos importantes son sus implicaciones: debido a que los supuestos y valores que conforman la ciencia reguladora condicionan el análisis del riesgo, tampoco podemos pensar que la solución al conflicto vaya a venir de la regulación fundamentada en la ciencia ( «science-based regulation»). Así, el principio de precaución también nos brinda la oportunidad de realizar la reflexión sobre los métodos de investigación y para ampliar el campo de conocimiento en la evaluación de los riesgos.

El principio de precaución en el proceso de evaluación da prioridad a la flexibilidad interpretativa. Además, su preferencia no se debe a la falta de conocimiento de los impactos negativos, sino al reconocimiento de que las evaluaciones son susceptibles de incorporar diferentes modelos de análisis, disciplinas, consideraciones y fuentes de preocupación. De esta manera, «la precaución, al reconocer y tratar de resolver lo desconocido, puede avanzar nuevos conocimientos. La oportunidad de considerar una amplia gama de posibilidades permite al proce- so precautorio ampliar su base cognitiva en la valoración del riesgo» (Stirling, 2003, 53). Podríamos entender el debate en torno a este principio de una manera diferente, si comprendemos los problemas que el proceso regulador nos ha mostrado a lo largo de los últimos años; en la evaluación tradicional del riesgo («science-based risk assessment») se detectan dos principales inconvenientes: tiene dificultades de integrar los efectos sistémicos a largo plazo e infravalora el rol que desempeñan los valores científicos.

Los conflictos relativos al riesgo se convierten en políticas de conocimiento; en parte, es un conflicto sobre las maneras de entender la incertidumbre científica. En este sentido, la precaución es el principio $\mathrm{y}$, sobre todo, la práctica que nos avanza la oportunidad de justificar e investigar esas incertidumbres (véanse COMEST, 2005; Myers y Raffensperger, 2006). Un ejemplo de las políticas de conocimiento, lo hallamos en la controversia sobre los efectos potenciales de los organismos genéticamente modificados. Por un lado, los testimonios de los biólogos moleculares y los ecologistas plantean diferentes conocimientos, cuestiones y preocupaciones. Por otro lado, el campo natural de estos conflictos no es el laboratorio: el conocimiento real se alcanza interviniendo en la naturaleza y a través de su transformación, y los conflictos dentro de la comunidad de expertos tiene relevancia social y su espacio real es más bien la esfera pública. Por tanto, la precaución no es una postura que cabe tomar ante una situación de incertidumbre, ni un principio para prevenir una actividad, sino aquel principio que nos motiva a considerar nuevos conocimientos, datos contrapuestos e incertidumbres en el proceso de evaluación y gestión de una innovación; esto también supone la oportunidad de analizar diferentes for- 
mas de gobierno y líneas de innovación alternativas (véanse Parson, 2001; Saunier y Meganck, 2007; Voß et alia, 2006). Precisamente, esa hipótesis es la propuesta alternativa que nos puede adelantar una interpretación diferente del principio de precaución dentro del nuevo contexto regulador.

El principio de precaución saca a la luz los límites del conocimiento científico que podamos estar manejando; por lo tanto, no sólo tendría implicaciones morales. Es decir, el tema de discusión se plantea de manera incorrecta, si tomamos como único tema de discusión la relación entre el comportamiento responsable y la posición moral; en otras palabras: el debate de si el cimiento de toda acción moral debe ser el conocimiento científico y, por lo tanto, debemos actuar de modo prudente en aquellos casos en los que debido a la estimación de los efectos negativos resulta aceptable la no-acción. Es éste el modo como la Comisión Europea propone actuar. No obstante, la propuesta alternativa del principio de precaución integra en sus normas de acción las preocupaciones epistemológicas relativas a la seguridad (véanse: Cranor, 2005; Raffensperger y deFur, 1999; Saner, 2002).

Esto ubica a los científicos y reguladores en una nueva situación: su preocupación no se limita a dar a conocer los riesgos previstos a través del proceso de evaluación, sino que han de confesar también las incertidumbres y los desconocimientos científicos descubiertos durante el análisis (algo que, como avanzan los estudios sobre percepción social de la ciencia, resulta parte de la reclamación ciudadana). Al fin y al cabo, la ciencia reguladora también es un ejercicio político implícito. Ciertamente, no podríamos entender la incertidumbre sobre los riesgos como un desconocimiento provisional: el conocimiento que manejamos siempre es incompleto, nuestro conocimiento puede contener incertidumbres y los tipos de interpretación pueden variar según los métodos científicos $\mathrm{y}$, por lo tanto, esos tipos de incertidumbre en última instancia dependen de una decisión de integrarlos a la política de investigación y regulación.

Esto acarrea dos consecuencias, que podemos identificarlos al comparar con el modelo tradicional de evaluación de los riesgos: en primer lugar, un desacuerdo científico entre expertos indicaría incertidumbre - $\mathrm{y}$, en su caso, ignorancia - sobre los riesgos; en segundo lugar, en situaciones de incertidumbre sobre los efectos potenciales, cabe optar por la precaución, incluso sin establecer un vínculo causal directo sobre los efectos potenciales. Esas medidas se corresponden con el nivel de protección acordado, es decir, según las normas establecidas sobre los efectos no tolerados; habría una interacción entre la previsión y la incertidumbre normativa, puesto que la incertidumbre científica estaría asociada a la incertidumbre que consideramos tolerable. De la misma manera que los diferentes métodos, modelos y disciplinas científicas ofrecen modos alternativos para entender la incertidumbre, también hay que decidir el tipo de incertidumbre que aceptamos. Esto supone la revisión de un supuesto que domina en la temática del riesgo, a saber, que la ciencia y los expertos, a través de las leyes de racionalidad y reglas normativas, garantizan el conocimiento verdadero y que su capacidad de control y predicción representa la certidumbre como garantía de la actividad científica y del proceso regulador.

Para concretar la magnitud de la propuesta, por tanto, la incertidumbre depende de aquello que nos preocupa y tratamos de evaluar. Es así como se entiende que «es la precaución la que justifica la incertidumbre, y no viceversa» (Levidow, 2001b); los conflictos sobre riesgo 
también se pueden entender así: es decir, como un conflicto entre las diferentes maneras de entender la incertidumbre científica relevante. La precaución, por lo tanto, sirve para plantear nuevas cuestiones y para identificar lo que nos resulta desconocido. Asimismo, la delimitación de un problema también enmarca las incertidumbres y desconocimientos del futuro, ya que aquello que no consideremos relevante también queda excluido del proceso de evaluación $\mathrm{y}$, por consiguiente, de ser identificado, estimado y valorado (Funtowicz et alia, 2000).

En este sentido, en el debate del riesgo también tienen lugar las diferentes interpretaciones del principio de precaución. Según esta propuesta, las discusiones relativas al principio de precaución podrían ser un indicador de los diferentes modos cognitivos para entender la incertidumbre. Así, los métodos científicos, provistos de valores, al promover diferentes tipos de conocimiento relevante, también se convierten en objeto de debate; si las evaluaciones del riesgo cuestionan los supuestos sobre la seguridad, es la evidencia de la incertidumbre la que toma centralidad en los discursos científicos y sociales. No se trata, pues, que en situaciones de incertidumbre su gestión posibilite estrategias políticas y morales diferentes, como se afirma en el contexto europeo ("la aplicación del principio de precaución forma parte de la gestión del riesgo cuando la incertidumbre cientifica no permite una evaluación completa del riesgo y cuando los responsables consideran que el nivel elegido de protección del medio ambiente o de la salud humana, animal o vegetal puede verse amenazado») (CEC, 2000, 13). Antes bien, las políticas de conocimiento conllevan la consideración de distintas incertidumbres y modos de entenderlas; cuando los métodos de investigación del riesgo y los conocimientos disponibles no son suficientes, los debates sobre el riesgo se con- vierten en un conflicto sobre los modos de entender la incertidumbre científica relevante. El principio de precaución no es, por lo tanto, la respuesta que una gestión responsable del riesgo proporciona a una evaluación del riesgo incompleta.

Es así como se modifica también el debate referido a la carga de la prueba, en tanto que nuestra definición alternativa de la precaución da a entender que existe un conflicto entre las diferentes maneras de conocer las incertidumbres más complejas. Con todo, el debate sobre los métodos científicos supone un conflicto entre los modos de conocer la incertidumbre. Ciertamente, cuando se discute sobre la precaución, existe un problema previo: la concreción de lo que resulta problemático y requiere un análisis científico. Desde el momento en que el análisis del riesgo acepta la precaución, se adapta la noción tradicional de la prueba científica (Wynne, 1992, 114); incorpora al debate un conflicto que es previo a la evaluación misma y es indicador de un desacuerdo más profundo: el conflicto no radica sólo en dar diferentes respuestas a las mismas preguntas, sino en establecer de modo distinto la agenda de problemas.

Así debemos entender, precisamente, la ambigüedad que a menudo se le reprocha al principio de precaución (Stirling, 1999): ya que el principio de precaución puede dejar al descubierto incertidumbres e ignorancias durante la evaluación del riesgo, el marco de supuestos para acordar y evaluar las causas y evidencias de los daños potenciales, se aleja de la certidumbre que ha caracterizado la ciencia moderna. Además, emergerían de forma más clara las dificultades epistemológicas que la precaución pueda presentar, si añadimos que no hay modo de conocer aquello que desconocemos. La condición de posibilidad del análisis del riesgo ha sido la certidumbre y ha sido posible establecer las implicaciones prácticas de 
una manera segura y concreta, pero el principio de precaución de hecho también cuestiona el supuesto de partida. Son estas dificultades las que hacen de la precaución un principio ambiguo, pero de una manera diferente a la que afirman los críticos del principio.

Por lo tanto, al delimitar el desconocimiento y la indeterminación, la función que desempeñan los valores y decisiones de los científicos y expertos se vuelve fundamental, y deja al descubierto los problemas de la «versión definitiva» de lo que se ha calificado como ciencia fundamentada. La incertidumbre científica y técnica puede acarrear el conflicto cultural en torno al riesgo, tal y como lo demuestra el debate tradicional sobre el principio de precaución. Pero existe una controversia más profunda: que la incertidumbre se puede fijar e interpretar según la consideración de los diferentes valores que manejan los científicos, expertos y políticos. El único debate de los valores y decisiones no se limita a discutir la viabilidad que ha de tener una línea de innovación o un proyecto tecnológico en situaciones de incertidumbre; eso hace que el tema de discusión se enfoque en la manera de interpretar la incertidumbre. No obstante, el conflicto de valores y decisiones dependen de la actividad reguladora: a la hora de analizar la incertidumbre también se integran los valores, no sólo durante la interpretación de hechos y datos, sino al acordar los hechos y datos a analizar. Así, la incertidumbre plantea de modo diferente lo que debemos comprender como conocimiento adecuado y relevante: en relación a los objetivos prácticos acordados, qué es razonable conocer, evaluar y regular; por lo tanto, la concreción de lo que es necesario conocer está rodeado de valores, no se trata únicamente de que las acciones se interpretan de una manera o de otra debido a la función social que puedan desempeñar los valores que motivan a los científicos.

El concepto de la precaución se ha entendido como una medida preventiva conforme a los riesgos que puede suponer un proyecto; en este sentido, sus promotores lo han concebido como una medida provisional, condicionada a la adquisición de nuevos conocimientos que permitan analizar las probabilidades y magnitud del impacto. Sin embargo, hemos visto que la precaución puede entenderse como una alternativa al análisis del riesgo en el proceso de regulación; antes de nada, la precaución llega a plantear un modo de hacer y regular la ciencia: plantea debatir los marcos cognitivos, las líneas y cuestiones a investigar, el sentido de la evidencia y el conocimiento adecuado.

\section{c. Actividades científicas y motivaciones políticas}

Existen diversas interpretaciones en torno al principio de precaución, pero no menos importante es considerar las críticas mutuas entre las diversas posiciones en el momento que el principio se convierte en objeto de competencia política: se establece una demarcación entre ciencia y política, y se argumenta que la posición del otro viene motivada por intereses políticos; hay un choque entre ciencia y política («science versus politics») y es la posición propia la que utiliza en sus análisis la ciencia fundamentada («sound science»). ${ }^{11}$ En este trabajo se ha propuesto una manera alternativa para entender los conflictos asociados a la precaución; hay un conflicto relativo a los estándares de regulación, y el principio de precaución no es más que un indicador de las diferentes maneras existentes de entender el problema del riesgo y el criterio de evidencia (Levidow et alia, 2005b, 273). 
Hay un conflicto sobre lo que consideramos incertidumbre relevante, riesgo tolerable y conocimiento adecuado. En este sentido, es cierto que la incertidumbre supone un debate sobre la viabilidad de una innovación e indica la existencia de diferentes posiciones culturales y políticas, pero hay otra interpretación más relevante: las incertidumbres científicas también dependen de los valores y supuestos que los científicos integran y la función que aquéllos juegan en el proceso de regulación (Levidow et allia, 2005a, 11).

La evaluación del riesgo depende de los criterios acordados para la regulación política, al ser ésta la que regula la lista de los impactos destacables, los criterios de evidencia y la consideración que merecen las incertidumbres científicas. Igualmente, los supuestos que enmarcan el conocimiento relevante estructuran las evaluaciones del riesgo (Millstone et allia, 2004); es lo que da a entender el principio de precaución, que adquiere sentido una vez reconocemos los problemas que tiene el análisis tradicional del riesgo (Levidow, 2001b, 868).

Toda declaración realizada por los expertos presupone una evaluación política del riesgo. En este sentido, la precaución supone un modo de interpretar los problemas que merecen ser identificados y de concebir la responsabilidad ante los impactos, de manera que incorpora un juicio normativo para interpretar si un conocimiento es aceptable. Esta crítica demuestra que es una diferenciación errónea el separar la regulación científica y la precaución. No obstante, en función de los problemas a identificar y la concepción del riesgo que integremos en el proceso de regulación para el análisis de los impactos, puede resultar que la precaución sea un principio que motive una actividad más científica y socialmente orientada (Stirling, 1999, 38).
Merece considerar esta interpretación alternativa, que ilustra sus implicaciones en el caso de los alimentos genéticamente modificados (Levidow et allia, 2005a): por una parte, en el momento de enmarcar las incertidumbres los expertos manejan una interpretación del principio de precaución; por otra parte, la precaución y los conflictos políticos son elementos constitutivos de la evaluación del riesgo y pueden resultar determinantes al acordar los criterios de evidencia a utilizar, los efectos no-deseados a identificar y las medidas de control a considerar; y, por último, el debate sobre los impactos ambientales también es un conflicto político sobre diferentes maneras de concebir modelos y alternativas de desarrollo agrícola. 12

Es así como debemos entender los diferentes conflictos, también aquéllos relativos a la oposición social. Y, por el contrario, las diferentes posiciones institucionales y sus respectivas críticas deben situarse más bien en un modo concreto de entender la actividad reguladora.

\section{Conclusiones generales}

En los últimos años, se han hecho notorias las disputas en torno a los estándares de regulación; una muestra corresponde al conflicto en torno al principio de precaución. No obstante, en todas ellas parece que el principio equivale a prevención y las críticas a la posición antagonista parten de la demarcación entre ciencia y política, de manera que la diferencia entre las posturas está motivada por los intereses políticos del contrario. En este trabajo he propuesto realizar una crítica a la ciencia reguladora y los supuestos que presupone la evaluación tradicional del riesgo: si bien es cierto que existen diferentes posiciones en torno a la precaución, todas ellas asumen la imagen corriente de la ciencia y el proceso de evaluación, y a falta de un modelo más 
sofisticado de la regulación también queda por analizar la precaución y, a partir de las reglas, criterios y procedimientos que de ella pueden emerger, también las acciones que genera en la acción científica y tecnológica.

El principio de precaución -en el contexto europeo - se ha definido y estructurado en la marco del análisis tradicional del riesgo; más concretamente, se sitúa en el lado de la gestión del riesgo, y las normas para la acción de la precaución tienen sentido una vez que la fase de evaluación reconoce que el conocimiento disponible imposibilita definir, estimar y valorar el riesgo. El principio de precaución, en todo caso, corresponde al ámbito político y es provisional. Las diferencias entre las distintas posiciones se deben a la manera de interpretar la función política del principio; si unos creen que la precaución viene motivada por la aversión al riesgo y la nueva cultura de la seguridad, otros creen que en algunos casos los límites del conocimiento y la incertidumbre sobre los riesgos justifican de modo provisional aplicar la precaución, prevenir los efectos no deseados y postergar el desarrollo y aplicación del nuevo producto hasta tener nuevos conocimientos.

En el tercer apartado de este trabajo me he centrado en la ciencia reguladora y he identificado una serie de problemas que han emergido en el análisis del riesgo. En la regulación basada en la ciencia también existen valores científicos que cumplen una función social y acotan las diferentes alternativas posibles. Un buen indicador de esto es el conflicto sobre los estándares de regulación existente dentro de la comunidad de científicos y la experteza; podemos hablar de una evaluación política de los riesgos desde el momento que debemos valorar y elegir el criterio de evidencia, las categorías para definir los efectos no deseados y los daños a identificar, las normas para diferenciar las consecuencias positivas y negativas de la innovación y por tanto para definir los principios de utilidad y aplicabilidad, las medidas de control adecuadas, así como los modos para prevenir los impactos y las herramientas metodológicas para evaluar los riesgos. Estos diferentes elementos llegan a definir lo que consideramos conocimiento relevante, pero además en un contexto en el que, junto a las preocupaciones teóricas, sociales y pragmáticas que también generan principios de acción, siempre habrá espacios de ignorancia; las políticas de conocimiento amplían el ámbito de análisis y los espacios de posibilidad de las consecuencias no deseadas, procura la integración de diferentes posibilidades, entre las que destacan aquéllas que emergen inducidas por los riesgos y las incertidumbres, pero las mismas dimensiones cognitivas adquieren un carácter posibilista: ella misma depende también de las representaciones y contextos que generan espacios de posibilidad cognitiva.

Los supuestos que presupone la evaluación del riesgo han estado condicionados por la imagen corriente de la ciencia: se concibió la función de la ciencia como la cognición del mundo real que nos rodea y, por ello, el conocimiento científico se orientaba de modo instrumental a la captura del objeto conocido o por descubrir; en la medida que cambia nuestro centro de atención del mundo real a los mundos posibles que pueden emerger a través de la realización de nuevos fenómenos y nuevas realidades, el conocimiento depende de los contextos, representaciones, indeterminaciones y nuevos horizontes, pero de esta manera hemos de reconocer también que la profundización del saber amplía el dominio de lo posible, pero también las posibilidades de la incertidumbre. El principio de precaución nos puede ayudar al menos a reconocer que el mundo no es un campo de acciones 
dadas y estructuras determinadas, sino que un espectro de muchos campos, algunos de los cuales no resultan cognoscibles.

En definitiva, todo esto implica que aquello que consideramos como daños ambientales está condicionado a los modos de vida y sus alternativas; así por ejemplo, en el caso de los alimentos modificados genéticamente, el conflicto hace referencia a los modelos de desarrollo agrícola y nuestras formas de vida. En segundo lugar, los conflictos relativos a la precaución son conflictos sobre los estándares de regulación; el principio no debe considerarse como una variante excepcional del análisis del riesgo, sino que genera sus propias reglas, normas, procedimientos e indicadores sobre conocimiento relevante. $\mathrm{Y}$, en tercer lugar, desde el momento que los estándares de regulación son objeto de competencia política, esto indica que no hacemos uso de la precaución para prevenir los impactos negativos en contextos de incertidumbre e ignorancia, sino que en un contexto de controversia sobre los modos, objetivos y motivos de la regulación, la precaución genera sus propios principios de acción y define los riesgos aceptables, las incertidumbres a evaluar y el conocimiento relevante de manera más compleja y acorde a las percepciones sociales.

\section{BIBLIOGRAFÍA}

Applegate, J. S. (2000): «The Precautionary Preference: An American Perspective on the Precautionary Principle», Human and Ecological Risk Assessment, Vol. 6, No. 3, pp. 413-443.

ASHFORD, N. A. (2005): «Incorporating Science, Technology, Fairness, and Accountability in Environmental, Health, and Safety Decisions», $\mathrm{Hu}$ - man and Ecological Risk Assessment, Vol. 11, No. 1, pp. 85-96.

Cash, B. (2006): «United States Policy on European Integration: An Understandable but Strategic Error Since 1990», The European Journal (March-April), 3-18.

CEC (2000): «Communication from the Commission on the Precautionary Principle», COM (2000) 1 final.

COMEST [World Commission on the Ethics of Scientific Knowledge and Technology] (2005): «The Precautionary Principle», Paris, United Nations Educational, Scientific and Cultural Organization.

Cranor, C. F. (2005): «Some Legal Implications of the Precautionary Principle: Improving Information-Generation and Legal Protections», $H u$ man and Ecological Risk Assessment, Vol. 11, No. 1, pp. 29-52.

Deblonde, M. y Du Jardin, P. (2005): «Deepening a Precautionary European Policy», Journal of Agricultural and Environmental Ethics, Vol. 18, No. 4, pp. 319-343.

ESRC Global Environmental Change Programme (2000a): «Steps into Uncertainty: Handling Risk \& Uncertainty in Environmental Policy-Making», Special Briefing, No. 6, University of Sussex, Brighton.

ESRC Global Environmental Change Programme (2000b): «Risky Choices, Soft Disasters», University of Sussex, Brighton.

Funtowicz, S.; Shepherd, I.; WILKINSON, D. y RaVetz, J. (2000): «Science and Government in the European Union: A Contribution to the Debate», Science and Public Policy, Vol. 27, No. 5, pp. 327-336.

GODARD, O. (dir.) (1997): Le principe de précaution dans la conduite des affaires humaines, Paris, MSH-INRA. 
Goldstein, B. D. y Carruth, R. S. (2005): «Implications of the Precautionary Principle: Is it a Threat to Science?», Human and Ecological Risk Assessment, Vol. 11, No. 1, pp. 209-219.

Hathcock, J. M. (2000): «The Precautionary Principle: An Impossible Burden Of Proof For New Products», AgBioForum, Vol. 3, No. 4, pp. 255258.

Holm, S. y Harris, J. (1999): «Precautionary Principle Stifles Discovery», Nature, Vol. 400, No. 6743, p. 398.

JASANOFF, S. (2003): «In a Constitutional Moment: Science and Social Order at the Millennium», in Joerges, B. y Nowotny, H. (eds.): Social Studies of Science and Technology: Looking Back, Ahead, Yearbook of the Sociology of the Sciences 23, Dordrecht, Kluwer, 155-180.

Jonas, H. (1974): Philosophical Essays:

From Ancient Creed to Technological Man, Englewood Cliffs/NJ, Prentice Hall.

JorDAN, A. (1999): «The Implementation of EU Environmental Policy: A Policy Problem Without a Political Solution?», Environmental and Planning, Vol. 17, No. 1, pp. 69-90.

Jordan, A. (2005): Environmental Policy in the European Union: Actors, Institutions and Processes, 2nd Edition, Oxford, Rowman \& Littlefield Publishers.

JoY, B. (2000): «Why the Future Doesn't Need Us», Wired 8.04, April 2000.

LeISS, W. (2005): «Policing Science: Genetics, Nanotechnology, Robotics», in Stehr, N. (ed.): Knowledge Politics: Governing the Consequences of Science and Technology, Boulder, Colorado, Paradigm Publishers, pp. 103116.

LEMKow, L. (2002): Sociología ambiental: pensamiento socioambiental $y$ ecología social del riesgo, Barcelona, Icaria.

LEvidow, L. (2001a): «Unsound Science? Trans-Atlantic Regulatory Disputes over GM Crops», National Europe Centre Papers, No. 3.

LEVIDOW, L. (2001b): «Precautionary Uncertainty: Regulating GM Crops in Europe», Social Studies of Science, Vol. 31, No. 6, pp. 842-874.

LEVIDOW, L.; CARR, S. y WIELD, D. (2005a): «EU Regulation of AgriBiotechnology: Precautionary Links between Science and Policy», Quality of Life and Management of Living Resources, Key Action 111-13, SocioEconomic Studies, Project n. ${ }^{\circ}$ QLRT2001-0034.

LeVidow, L.; CARr, S. y Wield, D. (2005b): «European Union Regulation of Agri-Biotechnology: Precautionary Links between Science, Expertise and Policy», Science and Public Policy, Vol. 32, No. 4, 261276.

Lujan, J. L. y TodT, O. (2007): «Precaution in Public: The Social Perception of the Role of Science and Values in Policy Making», Public Understanding of Science, Vol. 16, No. 1, pp. 97-109.

Mayer, S. y Stirling, A. (2002): «Finding A Precautionary Approach to Technological Developments: Lessons for the Evaluation of GM Crops», Journal of Agricultural and Environmental Ethics, Vol. 15, No. 1, pp. 52-71.

Miller, H. I. y ConKo, G. (2000): «The Science of Biotechnology Meets the Politics of Global Regulation», Issues in Science and Technology, Vol. 17, No. 1, pp. 47-54.

Miller, H. I. y Conko, G. (2001): «The Protocol's Illusionary Principle», $\mathrm{Na}$ ture Biotechnology, Vol. 18, pp. 360361. 
Millstone, E. et al. (2004): Science in Trade Disputes Related to Potential Risks: Comparative Case Studies, Luxembourg, Office for Official Publications of the European Communities.

Murphy, J. (2006): Governing Technology for Sustainability, Oxford, Rowman \& Littlefield Publishers.

Myers, N. (2002): «The Precautionary Principle Puts Values First», Bulletin of Science, Technology \& Society, Vol. 22, No. 3, pp. 210-219.

Myers, N. y RAFFensperger, C. (2006): Precautionary Tools for Reshaping Environmental Policy, Massachusetts, MIT Press.

PARSON, E. (ed.) (2001): Governing the Environment: Persistent Challenges, Uncertain Innovations, Toronto, University of Toronto Press.

Pieterman, R. (2001): "Culture in the Risk Society: An Essay on the Rise of a Precautionary Culture», Zeitschrift für Rechtssoziologie, Vol. 22, No. 2, pp. 145-168.

RAFFENSPERGER, C. y BARRETT, K. (2001): «In Defense of the Precautionary Principle», Nature Biotechnology, Vol. 19, p. 811.

RAFFENSPERGER, C. y Defur, P. L. (1999): «Implementing the Precautionary Principle: Rigorous Science and Solid Ethics», Human and Ecological Risk Assessment, Vol. 5, No. 5, pp. 933-941.

RENN, O. (2007): Risk Governance: Coping with Uncertainty in a Complex World, Oxford, Rowman \& Littlefield Publishers.

RenN, O. y Klinke, A. (2001): «Risk Evaluation and Risk Management for Institutional and Regulatory Policy», in Stirling, A. (ed.): On Science and Precaution in the Management of Technological Risk (vol. 2: Case Stu- dies), Seville, Institute for Prospective Technological Studies, pp. 11-35.

Sanderson, H. y Petersen, S. (2002): «Power Analysis as a Reflexive Scientific Tool for Interpretation and Implementation of the Precautionary Principle in the European Union», Environmental Science and Pollution Research, Vol. 9, No. 4, pp. 221-226.

SANER, M. (2002): «An Ethical Analysis of the Precautionary Principle», International Journal of Biotechnology, Vol. 4, No. 1, 81-95.

SAunier, R. E. y Meganck, R. A. (2007): Dictionary and Introduction to Global Environmental Governance, Oxford, Rowman \& Littlefield Publishers.

SILVER, L. M. (2006): Challenging Nature: The Clash of Science and Spirituality at the New Frontiers of Life, New York, Ecco/Harper Collins.

Stehr, N. (ed.) (2005): Knowledge Politics: Governing the Consequences of Science and Technology, Boulder, Colorado, Paradigm Publishers.

Stirling, A. (1999): «On "Precautionary" and "Science Based" Approaches to Risk Assessment and the Environmental Appraisal» (vol. 1: A Synthesis Report of Case Studies), Seville, Institute for Prospective Technological Studies.

STIRLING, A. (2003): «Risk, Uncertainty and Precaution: Some Instrumental Implications from the Social Sciences», in Berkhout, F.; LeACH, M. y SCOONER, I. (eds.): Negotiating Environmental Change: New Perspectives from Social Science, Cheltenham, Edward Elgar, pp. 33-76.

STIRLING, A. (2005): «Opening Up or Closing Down? Analysis, Participation and Power in the Social Appraisal of Technology», in LEACH, M.; SCOONER, I. y WyNnE, B. (eds.) 
(2005): Science and Citizens. Globalization \& the Challenge of Engagement, London, Zed Books, pp. 218231.

StiRling, A. (2007): «Risk, Precaution and Science: Towards a more Constructive Policy Debate: Talking Point on the Precautionary Principle», EMBO reports, Vol. 8, pp. 309-315.

Sunstein, C. R. (2003), «Beyond the Precautionary Principle», Public Law and Legal Theory Working Paper, n. ${ }^{\circ}$ 38, The Law School, The University of Chicago.

TÁbara, J. D.; Polo, D. y Lemkow, L. (2003): «Precaución, riesgo y sostenibilidad en los organismos agrícolas modificados genéticamente», Política y sociedad, Vol. 40, No. 3, pp. 81103.

Tickner, J. (ed.) (2003): Precaution, Environmental Science, and Preventive Public Policy, Washington, DC, Island Press.

TODT, O. (2002): Innovación y regulación: la influencia de los actores sociales en el cambio tecnológico. El caso de la ingeniería genética agrícola, Valencia, Universitat de Valencia (Tesis Doctoral).

VAN DEN BeLt, H. (2003): «Debating the Precautionary Principle: "Guilty until Proven Innocent" or "Innocent until Proven Guilty"?», Plant Physiology, Vol. 132, pp. 1122-1126.
VAN DEN BeLt, H. (2005): «Biotechnology, the US-EU dispute and the Precautionary Principle», in WESSELER, J. H. H. (ed.): Environmental Costs and Benefits of Transgenic Crops, Berlin, Springer, pp. 185-197.

Vogel, D. (1997): Barriers and Benefits: Regulation in Transatlantic Trade, Washington, DC, Brookings Institution Press.

Vogel, D. (2003): "The Hare and the Tortoise Revisited: The New Politics of Consumer and Environmental Regulation in Europe», British Journal of Political Science, Vol. 3, No. 4, pp. 557-580.

Voß, J-P.; BAUKnecht, D. y KemP, R. (eds.) (2006): Reflexive Governance for Sustainable Development, Cheltenham, Edward Elgar.

WYNNE, B. (1992): «Uncertainty and Environmental Learning: Reconceiving Science and Policy in the Preventive Paradigm», Global Environmental Change, Vol. 2, pp. 111-127.

WynNe, B. (2005): «Risk as Globalizing "Democratic" Discourse'? Framing Subjects and Citizens», in LEACH, M.; ScoOnes, I. y Wynne, B. (eds.) (2005): Science and Citizens: Globalization \& The Challenge of Engagement, London, Zed Books, pp. 66-82.

Wynne, B. y Meyer, S. (1993): «How Science Fails The Environment», New Scientists, 139 (1876): 33-35.
1 Véase Office of the United Status Trade Representative (2003): U.S. and Cooperating Countries File WTO Case Against EU Moratorium on Biotech Foods and Crops: EU's Illegal, Non-Science Based Moratorium Harmful to Agriculture and the Developing World. 13 May 2003.

2 Para explicar esta postura crítica, me sirvo de Applegate, 2000; Cash, 2006; Goldstein y Carruth, 2005; Miller y Conko 2000, 2001; van den Belt 2003, 2005.
3 Tal postura reproduce el dilema de Pascal acerca de la creencia en Dios. Véase: Van den Belt, 2003, 1123-1124.

4 Con la emergencia de los nuevos conflictos socio-técnicos y el principio de precaución hay un cambio radical ante la regulación a ambos lados del océano. La postura conservadora y reguladora estadounidense es sustituida por una interpretación más permisiva, y viceversa. Véanse Vogel, 1997, 2003. 
5 Para una exposición más amplia y exhaustiva, véanse Raffensperger y Barrett, 2001; Tickner, 2003.

${ }^{6}$ En realidad, existen dos posturas opuestas sobre el informe Brundtland, que está en el origen de los diferentes tratados internacionales sobre el ambiente: la «posición flexible» aprueba el informe, es decir, que la protección ambiental y el crecimiento económico son no sólo compatibles sino mutuamente dependientes. Sin embargo, la «posición fuerte» cuestiona ese programa analítico y político. Véanse Ashford, 2005; Deblonde y Du Jardin, 2005; Murphy, 2006.

7 Ante el supuesto de que las nuevas tecnologías (biotecnologías, nanotecnologías, robótica) pueden alterar los parámetros de definición de la existencia humana, se plantean medidas reguladoras que, además de restricciones y límites al desarrollo del conocimiento y el cambio tecnológico, suponen la prohibición (véase Joy, 2000). Con todo, ahora se habla de riesgos morales (más allá de riesgos técnicos) para la humanidad (véanse Leiss, 2005; Silver, 2006; Stehr, 2005); en lo que respecta a poner límites a nuestro conocimiento, resulta pertinente discutir y formular como principio moral «el derecho a la ignorancia» (Jonas, 1974, 161-163).

${ }^{8}$ A mi parecer, esta propuesta responde a las percepciones sociales sobre la precaución; véase Luján y Todt, 2007.

9 Es necesario hacer una distinción entre las diferentes categorías de incertidumbre. En primer lugar, el riesgo: es decir, la ciencia conoce las probabilidades y magnitud del daño. En segundo lugar, la ambigüedad: en este caso, la ciencia conoce las probabilidades, pero no está clara la magnitud del daño. En tercer lugar, la incertidumbre: es decir, a pesar de que se conoce el daño potencial, la distribución de las probabilidades no es segura. Por último, la ignorancia: es decir, existe incertidumbre hacia los dos componentes o se impone el desconocimiento. Esta división de los mo- dos de incertidumbre influye en el valor y credibilidad de la evaluación del riesgo. Ciertamente, el método tradicional, la evaluación probabilística del riesgo, puede proporcionar resultados adecuados y fidedignos sólo para la categoría del «riesgo», y de modo parcial para la noción de la «incertidumbre». No obstante, carece de capacidad de respuesta para las categorías «ambigüedad» e «ignorancia».

10 "[Las disputas inter-jurisdiccionales] no son tanto interpretaciones divergentes de los mismos datos, sino disputas asociadas a cuestiones politicas en la evaluación de riesgos como las diferentes líneas de investigación científica que deben ser desarrolladas, los efectos que han de ser considerados "nocivos", los tipos de evidencia que son necesarios y/o suficientes, todo ello antes por ejemplo de considerar los ensayos en las explotaciones agrícolas o cultivos comerciales como aceptablemente seguras» (Millstone et al., 2004, 18).

11 En este sentido, por ejemplo, en la actividad científica en general y en los procesos de evaluación y regulación en particular, se le ha denegado su papel al principio de precaución, "dado que por definición ;no tiene que basarse en la evidencia cientifica!», una «filosofia ambientalista» que pretende influir en los gobernantes y legisladores (véase Gray, 1990). No obstante, es el argumento que utilizan las diversas posiciones (véase la sección 2) para criticar a los oponentes, también por parte de la UE contra los partidarios de una interpretación fuerte del principio de precaución, a quienes se acusa de utilizar el principio motivado más bien por los desacuerdos que mantienen con el modelo social que promueve la tecnología y los motivos económicos que la inspiran (véanse Pieterman, 2001; Sunstein, 2003).

12 Veánse Myers, 2002; Tábara et allia, 2003. Desde esa suposición y sobre una gestión social de la ingeniería genética, véase Todt, 2002. 